\title{
EFECTO DE LOS ASPECTOS DEMOGRÁFICOS EN LA VALORACIÓN DE LA CALIDAD DEL SERVICIO. UNA REVISIÓN
}

\section{EFFECT OF THE DEMOGRAPHIC ASPECTS IN THE VALUATION OF THE QUALITY OF THE SERVICE. A REVIEW}

\author{
Juan Carlos Vergara Schmalbach ${ }^{1}$, Víctor Manuel Quesada Ibargüen ${ }^{2}$, Francisco Javier Maza Ávila ${ }^{3}$
}

\begin{abstract}
${ }^{1}$ Candidato a Doctor en Ciencias Sociales, mención Gerencia, profesor adscrito a la Facultad de Ciencias Económicas. Universidad de Cartagena (Colombia), Avenida del consulado Calle 30 ํo48 - 152, e-mail: jvergaras@unicartagena.edu.co; ${ }^{2}$ Doctor en Ingeniería de la Organización, profesor (jubilado). Universidad de Cartagena (Colombia), e-mail: vquesadaibarguen@gmail.com; ${ }^{3}$ Doctor en Ciencias Sociales y Jurídicas, profesor adscrito a la Facultad de Ciencias Económicas y al Instituto Internacional de Estudios del Caribe. Universidad de Cartagena (Colombia), Avenida del consulado Calle $30 \mathrm{~N}^{\circ} 48$ - 152, e-mail: fmazaa@unicartagena.edu.co
\end{abstract}

Rev. U.D.C.A Act. \& Div. Cient. 20(2): 457-467, Julio-Diciembre, 2017

\section{RESUMEN}

Comprender los elementos que determinan la satisfacción de usuarios o de clientes de un servicio permite a las organizaciones -tanto públicas como privadas- diseñar e implementar estrategias dirigidas a mejorar los niveles de lealtad y de recompra, entre otras intenciones futuras. Este artículo tiene como propósito evidenciar el efecto que tienen las variables demográficas, como elementos o constructos determinantes de la calidad de un servicio. Para lograr este cometido, se han tenido en cuenta artículos de investigación publicados en revistas indexadas en Scopus, Science Direct y Ebsco Host, durante el periodo 2006-2016, referentes a casos empíricos, donde se analizaron las relaciones de las variables demográficas sobre constructos, que caracterizan la calidad del servicio. La revisión permitió evidenciar la existencia de efectos, positivos o negativos, de los aspectos demográficos de los clientes o consumidores, sobre las expectativas, las percepciones, la satisfacción y las intenciones futuras, siendo el género, la edad, los ingresos y el nivel de estudios, las variables con mayores incidencias.

Palabras clave: Calidad del servicio, aspectos demográficos, satisfacción, intenciones futuras, percepción.

\section{SUMMARY}

Understanding the elements affecting the satisfaction of users and customers of a service enable organizations to conduct actions and strategies focused in performance the levels of loyalty and repurchase, among other future behaviour.
The purpose of this article is to evidence the demographic variables effect, as one of the factors or constructs which could become an important determinant of the service quality. To achieve this purpose were taken into account papers published in the last 10 years, that included into an empirical case, some demographics variables. As result from this analisys, was evidenced effects -positives or negativesfrom the demographics elements over the expectatives, perceptions, satisfaction and future behaviour, being the gender, age, income, and study level the variables with the most incidences.

Key words: Service quality, demographics, satisfaction, future Intentions, perception.

\section{INTRODUCCIÓN}

Los estudios referentes a la valoración de la calidad de un servicio permiten conducir a la identificación de los elementos que explican los niveles de satisfacción de un cliente, información coadyuvante en los procesos de toma de decisiones en las organizaciones, tanto públicas como privadas, con beneficio directo en dicho cliente (Vergara-Schmalbach et al. 2011; Vergara-Schmalbach \& Quesada, 2011). En la literatura, se encuentran diversos resultados de investigaciones que, mediante el desarrollo de aplicaciones empíricas, demuestran una relación consistente entre las expectativas y las percepciones -entendidas como antecedentes de la satisfacción-; también, señalan el papel de las percepciones, como motivadoras del comportamiento del cliente del servicio (Vergara-Schmalbach et al. 2012). Estas relaciones, se 
pueden representar bajo un modelo general de Ecuaciones Estructurales, que permite valorar los efectos entre los constructos que caracterizan el servicio (Bolton \& James, 1991).

El esquema general para el análisis de la calidad del servicio, según la simplificación propuesta por Cronin \& Taylor (1992), consiste en un modelo compuesto por tres factores o constructos: Percepción, Satisfacción e Intenciones futuras -este último, representado por variables como la lealtad, la recompra, las quejas y reclamaciones, la recomendación boca a boca y la recompra-; a su vez, dichos constructos son alimentados por un conjunto de datos, expresados a través de variables observadas, que provienen de una muestra o población.

La desventaja de la simplificación planteada con anterioridad, de amplia utilización en el análisis de la calidad de un servicio cualquiera, es que deja a un lado la posibilidad de introducir otros elementos que podrían ser determinantes de la calidad de un servicio, tales como los datos demográficos de los clientes. Vale anotar que las variables demográficas suelen estar presentes en los cuestionarios de percepción (Bradburn et al. 2004); sin embargo, su inclusión se limita únicamente a los siguientes aspectos:

- Indagar si una muestra está sesgada o es representativa de la población (Bradburn et al. 2004; Mitchell \& Jolley, 2010; 2012).

- Caracterizar la población encuestada, a partir de sus características demográficas (Mitchell \& Jolley, 2010).

- Definir nuevas variables independientes o predictores (Wedel \& Desarbo, 2002).

- Conocer el perfil del cliente o consumidor (Nettleton, 2003)

En este sentido, la inclusión de las características demográficas del cliente, no solo como una forma para facilitar filtros o clasificaciones -como suele ser consideradas-, sino como parte del modelo para evaluación de la calidad del servicio, podría traer, como principal beneficio, la posibilidad de contar con la información necesaria para el diseño de estrategias de segmentación. También permitiría la planeación de acciones dirigidas por grupos demográficos para mejorar los niveles de satisfacción y, por ende, la actitud favorable del cliente hacia el servicio en cuestión. Por ello, el objetivo de este artículo, se centra en evidenciar el efecto que tienen las variables demográficas, como elementos o constructos determinantes de la calidad de un servicio, a partir de la revisión de los artículos referentes a casos empíricos, publicados en revistas que se encuentren indexadas en las principales bases de datos, como Scopus, Science Direct y Ebsco Host, durante el periodo 2006-2016. Vale aclarar que una revisión previa durante el periodo de análisis no arrojó artículos de revisión similares al propuesto.
La estructura que recoge este artículo, se presenta a continuación: tras este epígrafe introductorio, en el siguiente apartado, se resume la metodología que se propone para la identificación de los artículos de investigación, referidos a casos empíricos, que consideran variables demográficas en el análisis de la calidad del servicio; en el apartado Resultados y Discusión, se analizan los métodos empleados, en cada uno de los artículos identificados, para el análisis cuantitativo del efecto de las variables demográficas en la calidad de un servicio; además, se muestran los efectos de dichas variables demográficas en la valoración de la calidad de un servicio. Al final de este apartado y de acuerdo con los resultados obtenidos, se presentan la Discusión y las Conclusiones. El último apartado corresponde a la Bibliografía.

\section{MATERIALES Y MÉTODOS}

Como propósito del presente artículo, se realizó una revisión de investigaciones sobre valoración de la calidad en distintos servicios que integran elementos del perfil demográfico de clientes, con el fin de demostrar, desde la literatura, evidencias del efecto que tienen las variables demográficas en la calidad del servicio, cómo se estructura este tipo de investigación y cuáles son los métodos que han empleado para su análisis. Para ello, se revisaron, de forma sistemática, artículos científicos indexados en las bases de datos especializadas Science Direct, Scopus y Ebsco Host, durante el periodo 2006-2016, orientados a demostrar la relación de las variables demográficas sobre otros constructos, sea a partir del desarrollo de un caso empírico o desde su aplicación a un servicio en particular. Par ello, se utilizaron las siguientes palabras clave, que fueron incorporadas a las bases de datos de forma combinada: Service Quality, Estructural Equation Modelling -SEM-, Demographics Variables, Gender, Age e Income. Para facilitar la inclusión y el análisis de los documentos consultados, se propuso un esquema de clasificación de las investigaciones, según el resultado arrojado, tal como se explica a continuación:

- Supuesto A: Se demuestra cuantitativamente que existe una o varias relaciones -que pueden ser causales o noentre las variables demográficas y las variables que valoran la calidad del servicio: Expectativas, Percepciones, Satisfacción e Intenciones futuras.

- Supuesto B: Se encuentran puntuaciones más altas en uno o varios indicadores o variables que cuantifican la calidad del servicio, de acuerdo a las clasificaciones demográficas, sin llegarse a demostrar un efecto correlacional o causal.

En el primer supuesto (Supuesto A), la o las variables demográficas tienen un efecto relacional o causal en una o varias variables estructurales del modelo; por ejemplo, al aumentar la edad del usuario o cliente encuestado, la satisfacción sobre el servicio recibido disminuye. Para el segundo resultado 
(Supuesto B), no se demuestra la relación causal entre el resto de variables consideradas; verbigracia, que el promedio de los hombres califique con una puntuación más alta la calidad del servicio recibido que las mujeres, pero no se demuestre una correlación significativa entre las variables género y la calidad del servicio.

Los resultados de la revisión bibliográfica podrían evidenciar el efecto que tienen las variables demográficas en la calidad de un servicio, lo que justificaría la importancia y la necesidad de su inclusión a la hora de realizar estudios de este tipo, así como para determinar cuáles, de dichas variables, deben ser priorizadas al momento de diseñar estrategias, que apunten a mejorar los niveles de satisfacción e intenciones futuras de los clientes.

\section{RESULTADOS Y DISCUSIÓN}

La revisión de la literatura sobre casos empíricos de valoración de calidad del servicio, que incluyen variables demográficas, arrojó un total de 28 artículos científicos, evidenciando la existencia de pocas investigaciones sobre el tema (Salvador, 2007). En los artículos científicos analizados, los datos demográficos suelen provenir de preguntas estandarizadas, tales como la edad, el género, los ingresos, la raza y la educación (Lavrakas, 2008), siendo la variable género la más empleada en las investigaciones revisadas. Se observa, que dichas variables suelen ser medidas como categóricas, cuyas respuestas encajan en categorías secuenciales, exhaustivas y mutuamente excluyentes (Melnyk \& Morrison-Beedy, 2012; Randolph \& Myers, 2013). Si la variable considera solo dos categorías posibles, esta variable suele ser clasificada como categórica dicotómica o binaria, como ocurre en la categorización usual del género, variable que acepta solo dos posibles resultados: masculino o femenino (Beaudry \& Miller, 2016).

La relación entre las variables demográficas con aquellas que influyen en la valoración de la calidad del servicio suele ser contradictoria (Lee et al. 2011), donde dichas contradicciones se desprenden de las diferentes valoraciones encontradas sobre las distintas dimensiones del servicio; por ejemplo, Ladhari \& Leclerc (2013) no pudieron demostrar una relación entre el género y la valoración de la calidad de los servicios financieros online, pero sí encontraron que las mujeres reportaron mayores niveles en la calidad del servicio, satisfacción, confianza y lealtad que los hombres. Por su parte, Zalatar (2012) realizó un estudio del servicio de bancos comerciales, arrojando que los hombres tienden a valorar mejor otros aspectos, como la infraestructura y los materiales, que son visualmente atractivos, mientras que las mujeres aprecian mejor la preparación de los empleados, en cuanto al conocimiento necesario para responder a las preguntas de los clientes. En línea contraria a las investigaciones anteriores, Ganesan-Lim et al. (2008), Tolpa (2012),
Belanche et al. (2016) y Zeng et al. (2016) no encontraron evidencia del efecto del género en la calidad del servicio.

La edad también es una variable demográfica de uso recuente en los estudios sobre valoración de la calidad. Ganesan-Lim et al. (2008) demostraron una fuerte evidencia entre la edad y las percepciones en la calidad del servicio, donde las valoraciones sobre lo tangible, los resultados y las interacciones fueron significativamente más altas en personas maduras comparadas con personas jóvenes.

Si las variables demográficas muestran un efecto significativo sobre las variables que cualifican la calidad del servicio en los estudios de percepción, se podría conducir estrategias para diferenciar o segmentar el servicio, a partir de la identificación del perfil de los usuarios (Khosrow-Pour, 2012; Wedel \& Desarbo, 2002). Es así que Calabrese et al. (2016) encontraron diferencias en cuanto a las expectativas y las percepciones sobre las acciones de Responsabilidad Social Empresarial llevadas a cabo por bancos, donde las mujeres puntuaron las expectativas significativamente más altas que los hombres; por tanto, en emprendimientos futuros, estas empresas podrían aumentar la eficacia, si en el diseño, la planificación, la ejecución y la evaluación de las iniciativas de Responsabilidad Social Empresarial tienen en cuenta dichas variaciones en las expectativas, según el género.

En cuanto a los servicios públicos, se registra en la literatura varios estudios que consideran las variables demográficas, dentro del análisis de la calidad de los servicios ofrecidos, con resultados ambiguos; por ejemplo, en los resultados del estudio realizado por Zeng et al. (2016) sobre el servicio de recolección de basuras, no se encontró un efecto significativo del género sobre la disposición a pagar por el servicio. Por otro lado, Morton et al. (2016) hallaron que, al valorar la calidad del servicio de transporte, los hombres tienden a calificar un poco mejor que las mujeres, los aspectos relacionados con la seguridad. En un análisis ampliado, basado en las 5 dimensiones expuestas por Parasuraman et al. (1988), Mokhlis (2012) encontró un efecto significativo en las dimensiones Tangibles y Confiabilidad, donde las mujeres tienden a otorgar una importancia mayor en estos dos elementos que los hombres, en el momento de evaluar la satisfacción total sobre el servicio recibido.

En la misma línea, Belanche et al. (2016) no pudieron demostrar una relación directa entre el género, el apego a la ciudad y el uso de los servicios públicos urbanos, explicado por la naturaleza propia de los servicios públicos, que están orientados a toda la comunidad; sin embargo, en ese estudio, la edad y el nivel de educación tuvieron un efecto positivo y negativo sobre el apego a la ciudad, respectivamente. En la tabla 1, se resumen los estudios analizados, clasificados cronológicamente, según el tipo de resultado documentado y el efecto generado 
Tabla 1. Estudios analizados sobre el efecto del género en la percepción de la calidad.

\begin{tabular}{|c|c|c|c|}
\hline Autores del estudio & $\begin{array}{l}\text { Tipo de } \\
\text { resultado }\end{array}$ & $\begin{array}{l}\text { Variables con efecto } \\
\text { significativo }\end{array}$ & Descripción del Efecto \\
\hline Anic \& Radas (2006) & Supuesto A & $\begin{array}{l}\text { Género, Tamaño del Hogar, } \\
\text { Edad, Localización }\end{array}$ & $\begin{array}{l}\text { Las mujeres muestran ser más leales que los hombres. Las } \\
\text { personas más cercanas al sitio de venta son más leales que } \\
\text { las personas lejanas. La edad y el tamaño del hogar mues- } \\
\text { tran una relación positiva con la lealtad del cliente. }\end{array}$ \\
\hline Salvador (2007) & Supuesto A & Género & $\begin{array}{l}\text { Resultados variados sobre la puntuación de los indicadores } \\
\text { de calidad, expectativas e indicadores de satisfacción. }\end{array}$ \\
\hline Patterson (2007) & Supuesto A & Edad, Ocupación & $\begin{array}{l}\text { Las personas con edad entre } 35 \text { años o más muestran un } \\
\text { comportamiento más leal que los clientes más jóvenes. }\end{array}$ \\
\hline $\begin{array}{l}\text { Ganesan-Lim et al. } \\
(2008)\end{array}$ & Supuesto A & Edad & $\begin{array}{l}\text { La edad tiene un efecto positivo en todos los aspectos de } \\
\text { la calidad del servicio. }\end{array}$ \\
\hline Juwaheer (2011) & Supuesto A & Género & $\begin{array}{l}\text { Las mujeres encuestadas evaluaron las expectativas con un } \\
\text { puntaje promedio mayor a los hombres en todas las vari- } \\
\text { ables de calidad. En cuanto a las percepciones, las mujeres } \\
\text { mostraron una puntuación en } 5 \text { de } 7 \text { variables. }\end{array}$ \\
\hline Palli \& Mamilla (2012) & Supuesto A & Género & $\begin{array}{l}\text { Los resultados indicaron que no hubo diferencias significa- } \\
\text { tivas en la valoración de la satisfacción general de los en- } \\
\text { cuestados en cuanto a la edad, la ocupación y el ingreso. } \\
\text { Se encontró diferencia significativa en la satisfacción gen- } \\
\text { eral de los encuestados sólo en términos de género }\end{array}$ \\
\hline $\begin{array}{l}\text { Anand \& Selvaraj } \\
(2012)\end{array}$ & Supuesto A & Localización & $\begin{array}{l}\text { Este estudio reveló que no existe una relación significativa } \\
\text { entre las variables demográficas y el nivel de satisfacción, } \\
\text { con excepción a la elección de la entidad financiera con } \\
\text { respecto a la localización. }\end{array}$ \\
\hline Mokhlis (2012) & Supuesto A & Género & $\begin{array}{l}\text { Las mujeres tienen una incidencia mayor que los hombres } \\
\text { en la puntuación de la satisfacción a partir de las valora- } \\
\text { ciones de los tangibles, fiabilidad sobre el servicio recibido. }\end{array}$ \\
\hline Zalatar (2012) & Supuesto B & Género & $\begin{array}{l}\text { Resultados variados sobre la puntuación de expectativas y } \\
\text { percepciones. }\end{array}$ \\
\hline Tolpa (2012) & Supuesto B & Género & $\begin{array}{l}\text { No existe mucha diferencia entre los géneros masculino y } \\
\text { femenino sobre las expectativas en el servicio. }\end{array}$ \\
\hline Sasikala (2013) & Supuesto A & $\begin{array}{l}\text { Género, Edad, Estado Civil, } \\
\text { Ocupación, Ingreso }\end{array}$ & $\begin{array}{l}\text { Demuestran una asociación significativa entre género, } \\
\text { edad, estado civil, ocupación y la calidad del servicio, sat- } \\
\text { isfacción y lealtad. No demuestran una asociación entre } \\
\text { ingreso y satisfacción. No encuentran una asociación sig- } \\
\text { nificativa entre el nivel de educación y la calidad del servi- } \\
\text { cio, satisfacción y lealtad. }\end{array}$ \\
\hline $\begin{array}{l}\text { Maruvada \& Bellam- } \\
\text { konda (2013) }\end{array}$ & Supuesto A & Género, Ingreso & $\begin{array}{l}\text { Existe un efecto entre el género y el ingreso el servicio ofre- } \\
\text { cido abordo. }\end{array}$ \\
\hline Akbar (2013) & Supuesto A & Género, ocupación, ingreso & $\begin{array}{l}\text { Las variables demográficas explican en un } 57 \% \text { la variación } \\
\text { de la puntuación en la lealtad del cliente (intención de } \\
\text { re-compra), en un } 52 \% \text { la variación en la recomendación } \\
\text { boca a boca y } 54 \% \text { en la insinceridad sobre el precio. }\end{array}$ \\
\hline Saad et al. (2013) & Supuesto A & Ingreso & $\begin{array}{l}\text { El nivel de ingreso tiene un efecto positivo sobre la lealtad } \\
\text { del cliente (entre mayor es el ingreso, mayores son los } \\
\text { niveles de lealtad). }\end{array}$ \\
\hline Afzal \& Pakistan (2013) & Supuesto A & $\begin{array}{l}\text { Edad, Educación, Es- } \\
\text { tado Civil, Duración de la } \\
\text { Relación con la empresa, } \\
\text { Categoría del Cliente }\end{array}$ & $\begin{array}{l}\text { La edad, educación, duración de la relación con la empre- } \\
\text { sa y categoría del cliente tienen un impacto positivo en la } \\
\text { lealtad del cliente. Las personas casadas tienden a ser más } \\
\text { leales con la empresa que los solteros. }\end{array}$ \\
\hline
\end{tabular}


Continuación Tabla 1.

\begin{tabular}{|c|c|c|c|}
\hline Autores del estudio & $\begin{array}{l}\text { Tipo de } \\
\text { resultado }\end{array}$ & $\begin{array}{l}\text { Variables con efecto } \\
\text { significativo }\end{array}$ & Descripción del Efecto \\
\hline $\begin{array}{l}\text { Bhattacharya \& Dash } \\
\text { (2013) }\end{array}$ & Supuesto A & $\begin{array}{l}\text { Edad, Educación, Ocu- } \\
\text { pación, Ingreso }\end{array}$ & $\begin{array}{l}\text { La satisfacción global del cliente es dependiente de la } \\
\text { edad, educación, ocupación e ingreso del encuestado. }\end{array}$ \\
\hline Irshad et al. (2013) & Supuesto A & Edad. Ingreso, Educación & $\begin{array}{l}\text { El nivel de ingresos y la edad (establecida por grupos de } \\
\text { usuarios) tienen un impacto moderado en el clima del } \\
\text { servicio. El nivel de educación tiene un impacto en el clima } \\
\text { del servicio y la percepción sobre la calidad del servicio. }\end{array}$ \\
\hline Singh (2013) & Supuesto A & $\begin{array}{l}\text { Género, Educación, In- } \\
\text { greso, Ocupación }\end{array}$ & $\begin{array}{l}\text { El género tiene un efecto en algunos aspectos de la calidad } \\
\text { del servicio (capacidad de resolver problemas, motivación } \\
\text { de amigos y parientes a comprar). El nivel de educación, el } \\
\text { nivel de ingreso y estado civil tienen efectos diferentes en la } \\
\text { intención de comprar en otro almacén. El estado civil tiene } \\
\text { un efecto significativamente alto en el comportamiento de } \\
\text { las quejas de los clientes }\end{array}$ \\
\hline Jain (2013) & Supuesto B & $\begin{array}{l}\text { Educación, Género, Ocu- } \\
\text { pación, Ingreso }\end{array}$ & $\begin{array}{l}\text { Los clientes sin nivel universitario tienen puntuaciones } \\
\text { significativamente mayores que los clientes graduados con } \\
\text { respecto a la percepción de aseguramiento de la calidad } \\
\text { del servicio. Los clientes con menor nivel de ocupación e } \\
\text { ingresos valoran la calidad con una puntuación mejor. }\end{array}$ \\
\hline $\begin{array}{l}\text { Ladhari } \\
\text { \& Leclerc (2013) }\end{array}$ & Supuesto A & Género & $\begin{array}{l}\text { Las mujeres reportaron mayores niveles en la calidad del } \\
\text { servicio, satisfacción, confianza y lealtad que los hombres }\end{array}$ \\
\hline Min \& Khoon (2014) & Supuesto A & $\begin{array}{l}\text { Nacionalidad, Género, } \\
\text { Edad, Estudios }\end{array}$ & $\begin{array}{l}\text { Los hombres valoran con una puntuación más alta los in- } \\
\text { dicadores de motivación (expectativas y percepciones) y la } \\
\text { satisfacción. La nacionalidad, edad y nivel de estudios tiene } \\
\text { efectos variados en la valoración de la calidad. }\end{array}$ \\
\hline Mutlaq \& Otaibi (2014) & Supuesto A & $\begin{array}{l}\text { Edad, Tamaño de la Familia, } \\
\text { Ingreso }\end{array}$ & $\begin{array}{l}\text { La edad, el tamaño de la familia y el ingreso tienen un } \\
\text { efecto positivo sobre la calidad percibida del servicio. }\end{array}$ \\
\hline Hagan (2015) & Supuesto A & $\begin{array}{l}\text { Género, Ocupación, Edu- } \\
\text { cación }\end{array}$ & $\begin{array}{l}\text { Las mujeres perciben la calidad del servicio con una pun- } \\
\text { tuación mayor. Los clientes con menor nivel de educación } \\
\text { tienen una buena percepción de la calidad del servicio. Las } \\
\text { personas retiradas perciben la calidad del servicio como } \\
\text { alta. }\end{array}$ \\
\hline Zeng et al. (2016) & Supuesto A & Edad, localización, ingreso & $\begin{array}{l}\text { A mayor edad, mayor disposición para pagar, A mayor } \\
\text { ingreso, menor disposición para pagar. En cuanto a la } \\
\text { localización, a mejor ubicación o estatus, mayor es la dis- } \\
\text { posición para pagar por el servicio. }\end{array}$ \\
\hline Morton et al. (2016) & Supuesto A & $\begin{array}{l}\text { Edad, Género, Estatus In- } \\
\text { greso, Educación }\end{array}$ & $\begin{array}{l}\text { Existe evidencia del género con efecto positivo sobre la } \\
\text { calidad del servicio (Masculino = } 1, \text { Mujer = } 0 \text { ). También } \\
\text { se evidencia que, a mayor edad, mayor es la valoración de } \\
\text { la calidad del servicio. } \\
\text { Con respecto al estado económico, los autores evidencian } \\
\text { diversos efectos. También concluyen que a mejor nivel de } \\
\text { educación, menor es la valoración de la calidad del servi- } \\
\text { cio. }\end{array}$ \\
\hline Calabrese et al. (2016) & Supuesto B & Edad, Género, Educación & $\begin{array}{l}\text { Resultados variados sobre la puntuación de expectativas y } \\
\text { percepciones. }\end{array}$ \\
\hline Belanche et al. (2016) & Supuesto A & Edad, Educación & $\begin{array}{l}\text { La edad tiene un efecto positivo sobre la valoración de los } \\
\text { tangibles, mientras que la educación tiene un efecto con- } \\
\text { trario. }\end{array}$ \\
\hline
\end{tabular}

Fuente: Elaboración propia, a partir del análisis de diversos estudios sobre género y calidad del servicio. 
por las variables demográficas identificadas -Supuesto A o B-. Uno de los fines de los modelos cuantitativos de valoración de la calidad de un servicio cualquiera consiste en permitir la detección de relaciones significativas entre los constructos del modelo, para poder determinar los factores que tienen efecto sobre los indicadores de calidad. Para validar los constructos y poder encontrar las relaciones significativas, se emplean uno o varios métodos multivariados para el análisis de datos, tales como las Regresiones Múltiples, Análisis de Componentes Principales, Análisis Discriminante múltiple, Análisis de Correlaciones, Análisis de Varianza y Covarianza, Análisis de Clúster, Análisis Conjunto, Análisis de Correspondencia, Análisis Factorial Confirmatorio y Modelos de Ecuaciones Estructurales, entre otros (Hair Jr et al. 2014).
Para Steenkamp \& Van Trijp (1991), las relaciones entre variables independientes y dependientes, suelen ser representadas como hipótesis estadísticas dentro de un modelo conceptual, donde se prueba si existe un efecto significativo entre las variables demográficas sobre las demás variables, que caracterizan la calidad del servicio; la validez equivale al grado en que los constructos alcanzan un significado empírico y teórico. En la tabla 2, se muestran los métodos de análisis de datos en los estudios indagados sobre valoración de la calidad en servicios variados, tales como financieros, transporte, comercio, educación y salud, entre otros, que incluyen variables demográficas, dentro de sus modelos cuantitativos.

Tabla 2. Caracterización de los estudios analizados sobre casos de valoración de la calidad del servicio, que incluyen variables demográficas.

\begin{tabular}{|c|c|c|c|c|}
\hline Servicio evaluado & Autores del estudio & Variables demográficas & $\begin{array}{l}\text { Tamaño de } \\
\text { la muestra }\end{array}$ & Método / herramienta utilizada \\
\hline \multirow{9}{*}{ Financiero } & Saad et al. (2013) & $\begin{array}{l}\text { Género, Edad, Ingreso, } \\
\text { Ocupación, Estilo de Vida }\end{array}$ & 150 & Regresión Múltiple \\
\hline & Zalatar (2012) & Género & 96 & Análisis de brechas / GAP \\
\hline & $\begin{array}{l}\text { Ladhari \& Leclerc } \\
\text { (2013) }\end{array}$ & Edad, Género, Educación & 376 & $\begin{array}{l}\text { Modelos de Ecuaciones } \\
\text { Estructurales }\end{array}$ \\
\hline & Jain (2013) & $\begin{array}{l}\text { Educación, Género, } \\
\text { Ocupación, Ingreso }\end{array}$ & 330 & Correlación, Prueba F, ANOVA \\
\hline & Ramez (2011) & $\begin{array}{l}\text { Género, Edad, Educación, } \\
\text { Nacionalidad, Ocupación }\end{array}$ & 195 & ANOVA \\
\hline & $\begin{array}{l}\text { Anand \& Selvaraj } \\
(2012)\end{array}$ & $\begin{array}{l}\text { Género, Estado Civil, Edad, } \\
\text { Educación, Dependientes, } \\
\text { Ocupación, Ingresos }\end{array}$ & 50 & Prueba Chi-Cuadrado \\
\hline & Sasikala (2013) & $\begin{array}{l}\text { Género, Edad, Estado Civil, } \\
\text { Ocupación, Ingreso, } \\
\text { Educación }\end{array}$ & 200 & ANOVA / Análisis de correlaciones \\
\hline & $\begin{array}{l}\text { Afzal \& Pakistan } \\
\text { (2013) }\end{array}$ & $\begin{array}{l}\text { Género, Edad, Educación, } \\
\text { Estado Civil, Duración de } \\
\text { la Relación con la empresa, } \\
\text { Categoría del Cliente }\end{array}$ & 200 & Prueba Chi-Cuadrado / ANOVA \\
\hline & $\begin{array}{l}\text { Bhattacharya \& Dash } \\
\text { (2013) }\end{array}$ & $\begin{array}{l}\text { Género, Edad, Estado Civil, } \\
\text { Localización, Educación, } \\
\text { Ocupación, Ingreso }\end{array}$ & 518 & Prueba Chi-Cuadrado \\
\hline \multirow{3}{*}{ Servicios Públicos } & Belanche et al. (2016) & Edad, Género, Educación & 464 & $\begin{array}{l}\text { Modelos de Ecuaciones Estruc- } \\
\text { turales / Regresión de mínimos } \\
\text { cuadrados parciales (PLS) }\end{array}$ \\
\hline & Mokhlis (2012) & Edad, Género, Educación & 234 & $\begin{array}{l}\text { Análisis de Regresión Múltiple / } \\
\text { Análisis Discriminante } \\
\text { Multivariado }\end{array}$ \\
\hline & Zeng et al. (2016) & $\begin{array}{l}\text { Edad, Género, Educación, } \\
\text { Localización, Ingreso }\end{array}$ & 518 & Regresión logística \\
\hline
\end{tabular}


Continuación Tabla 2.

\begin{tabular}{|c|c|c|c|c|}
\hline Servicio evaluado & Autores del estudio & Variables demográficas & $\begin{array}{l}\text { Tamaño de } \\
\text { la muestra }\end{array}$ & Método / herramienta utilizada \\
\hline \multirow{4}{*}{ Transporte } & Tolpa (2012) & Edad, Género & 79 & Análisis de brechas / GAP \\
\hline & Morton et al. (2016) & $\begin{array}{l}\text { Edad, Género, Ingreso, edu- } \\
\text { cación }\end{array}$ & 3797 & Análisis Factorial Confirmatorio \\
\hline & $\begin{array}{l}\text { Ganesan-Lim et al. } \\
(2008)\end{array}$ & Edad, Género, Ingreso & 224 & Análisis Factorial Confirmatorio \\
\hline & $\begin{array}{l}\text { Maruvada \& } \\
\text { Bellamkonda (2013) }\end{array}$ & Género, Ingreso & 607 & MANOVA \\
\hline \multirow{3}{*}{ Comercio } & Anic \& Radas (2006) & $\begin{array}{l}\text { Género, Edad, Tamaño del } \\
\text { Hogar, Ingreso, Localización }\end{array}$ & 300 & Regresión Múltiple \\
\hline & Mutlaq \& Otaibi (2014) & $\begin{array}{l}\text { Género, Estado Civil, Edad, } \\
\text { Educación, Tamaño del Ho- } \\
\text { gar, Ingreso, Localización }\end{array}$ & 408 & Regresión Múltiple \\
\hline & Singh (2013) & $\begin{array}{l}\text { Género, Edad, Localidad, } \\
\text { Estado Civil, Educación, } \\
\text { Ocupación, Ingresos }\end{array}$ & 540 & ANOVA \\
\hline \multirow{3}{*}{ Turismo / Hotelería } & Hagan (2015) & $\begin{array}{l}\text { Nacionalidad, Edad, Género, } \\
\text { Educación, Religión, Ocu- } \\
\text { pación }\end{array}$ & 358 & ANOVA \\
\hline & Juwaheer (2011) & Género & 401 & $\begin{array}{l}\text { Prueba Chi-Cuadrado / MANOVA / } \\
\text { Análisis Discriminante } \\
\text { Multivariado }\end{array}$ \\
\hline & Akbar (2013) & $\begin{array}{l}\text { Género, Edad, Estado Civil, } \\
\text { Localización, Ocupación, } \\
\text { Educación }\end{array}$ & 680 & Regresión Múltiple \\
\hline \multirow{2}{*}{ Educación } & Min \& Khoon (2014) & $\begin{array}{l}\text { Nacionalidad, Género, Edad, } \\
\text { Estudios }\end{array}$ & 263 & $\begin{array}{l}\text { Modelos de Ecuaciones } \\
\text { Estructurales }\end{array}$ \\
\hline & Palli \& Mamilla (2012) & $\begin{array}{l}\text { Edad, Género, Ocupación, } \\
\text { Ingreso }\end{array}$ & 120 & Análisis de correlaciones / ANOVA \\
\hline \multirow{2}{*}{ Servicios varios } & Salvador (2007) & Edad, Género & 1008 & Análisis de Regresión Múltiple \\
\hline & Patterson (2007) & Género, Edad, Ocupación & 700 & ANOVA \\
\hline $\begin{array}{l}\text { Responsabilidad } \\
\text { Social Empresarial }\end{array}$ & Calabrese et al. (2016) & Edad, Género, Educación & 908 & Coeficiente de Cohen (d) \\
\hline Salud & Irshad et al. (2013) & $\begin{array}{l}\text { Género, Edad. Ingreso, Edu- } \\
\text { cación }\end{array}$ & 912 & ANOVA \\
\hline
\end{tabular}

Fuente: Elaboración propia, a partir del análisis de diversos estudios sobre calidad del servicio.

En las investigaciones consultadas, se desarrollaron casos empíricos, en su mayoría, centrados en un servicio en particular. En la tabla 3, se muestra una ficha resumen sobre los elementos usuales que estructuran las investigaciones analizadas.

En cuanto a la medición de las relaciones entre las variables demográficas y los demás constructos, es apropiado codificar las categorías dispuestas por variable demográfica para su posterior tratamiento en los modelos cuantitativos (David $\&$ Sutton, 2011). Una forma de codificación consiste en la asignación de un número consecutivo para cada una de las categorías de selección o a través de una agrupación particular de categorías, por ejemplo, convirtiendo las variables categóricas en categóricas dicotómicas, permitiendo su inclusión y su tratamiento dentro del modelo, como variables discretas (Monette et al. 2014) o como variables Dummy, en modelos de regresión (Hardy, 1993). En las variables nominales, por ejemplo, ocupación, el orden de la numeración asignado por categoría no es relevante (Rubin, 2013). En la tabla 4, se ilustra un ejemplo de la codificación de las categorías de variables demográficas. 
Tabla 3. Caracterización resumen de los estudios analizados sobre variables demográficas.

\begin{tabular}{|l|c|}
\hline Número de variables demográficas promedio & 4 \\
\hline Número de variables demográficas promedio con efecto significativo & 3 \\
\hline Variables demográficas con mayor número de efectos encontrados (Aproximado) & Género, Edad \\
\hline Tamaño de muestra promedio & 522,11 \\
\hline Tamaño de muestra mínimo & 79 \\
\hline Tamaño de muestra máximo & 3797 \\
\hline Método estadístico más utilizado & ANOVA \\
\hline
\end{tabular}

Fuente: Elaboración propia, a partir del análisis de diversos estudios sobre calidad del servicio analizados.

Tabla 4. Codificación de categorías demográficas segmentadas en dos opciones.

\begin{tabular}{|l|l|l|}
\hline \multicolumn{1}{|c|}{ Pregunta } & \multicolumn{1}{|c|}{ Codificación / Categoría } & Tipo de Variable \\
\hline Género & $\begin{array}{l}0-\text { Masculino } \\
1-\text { Femenino }\end{array}$ & Nominal, Dicotómica \\
\hline \multirow{5}{*}{ Edad } & $\begin{array}{l}0-15 \text { a } 29 \text { años } \\
1-30 \text { a } 54 \text { años }\end{array}$ & \\
& $2-55$ a 64 años & Intervalo \\
& $3-65$ a 79 años & \\
& $4-$ Mayores de 80 años & \\
\hline \multirow{5}{*}{ Ingreso } & $0-\$ 15000-25000$ & \\
& $1-\$ 26000-35000$ & Ratio \\
& $2-\$ 36000-50000$ & \\
& $3-\$ 50000-100000$ & \\
& $4-\$ 100000-250000$ & \\
& $5-$ Ingresos mayores a $\$ 250000$ & \\
\hline
\end{tabular}

Fuente: Elaboración propia, basado en la clasificación de categorías agrupadas realizada por Ganesan-Lim et al. (2008).

En cuanto al tratamiento cuantitativo de los aspectos demográficos en modelos correlacionales y causales, se deben considerar el carácter cualitativo de las variables nominales que, a pesar de una codificación, serán consideradas como discretas por naturaleza y, por tanto, no podrán ser asumidas como normalmente distribuidas (Finney \& Distefano, 2006) afectando un requisito de los modelos estadísticos de tipo paramétricos.

En resumen, y a partir del análisis de artículos de investigación referidos a la valoración de la calidad del servicio, se evidencia la existencia de diversos efectos de los aspectos demográficos de los clientes o consumidores sobre las expectativas, las percepciones, la satisfacción y las intenciones futuras. Con la información analizada en este artículo, se complementa el conocimiento general sobre el efecto de las variables demográficas, cuyo aporte principal radica en la justificación de la inclusión de los datos demográficos en los estudios de valoración de la calidad del servicio, en el desarrollo de nuevos casos.

Los resultados del análisis planteado en este apartado muestran que los efectos de las variables demográficas sobre la calidad del servicio son diversos; estas diferencias obedecen al tipo de servicio recibido, las dimensiones evaluadas y las actitudes entre géneros. Pueden, además, variar entre países, con contextos históricos y culturales diferentes, como concluyen los estudios realizados por Yoshida (2011) y Marković \& Janković (2013). En el caso particular de la variable Género, que la persona sea hombre o mujer, tendría un efecto en la forma de valorar el servicio recibido, lo que podría influenciar en el resultado final. Estos cambios en el esquema de pensamiento, se pueden deber a estereotipos (Ganesan-Lim et al. 2008), exposición hormonal, teorías socio-culturales, 
teoría de la evolución y la hipótesis de selectividad que, a su vez, definen la ética y la moralidad, la confianza, las emociones, la sensibilidad, el estilo paternal e, incluso, los comportamientos de consumo (Meyers-Levy \& Loken, 2015).

Como se demuestra en las investigaciones consultadas, la edad, el ingreso y el nivel de estudio, también pueden ser importantes determinantes del comportamiento del consumidor, al igual que el género; un ejemplo de este tipo de resultados, se encuentra en el estudio realizado por Hagan (2015), en el servicio de hoteles, donde el autor recomienda la inclusión de las variables nivel de educación y de ocupación, además del género, para mejorar la calidad del servicio, a partir de la segmentación por grupo de invitados.

El conocimiento de la existencia de incidencias de las variables demográficas sobre la calidad de un servicio no solo podría, sino que debería, derivar en la formulación e implementación de estrategias diferenciadas por grupos segmentados de individuos, enfocadas en mejorar los indicadores de satisfacción e intenciones futuras, adaptando los servicios a las características demográficas de los clientes o consumidores. Para Mokhlis (2012) y Min \& Khoon (2014) esta diferenciación ayudaría al desarrollo de estrategias de mercado basado en las variables demográficas, cuyos datos provendrían de las consultas realizadas a los clientes y el tratamiento cuantitativo, bajo esquemas que permitan determinar las relaciones entre variables estructurales, adecuadas al contexto. Como recomendación final, en futuras investigaciones sobre estrategias empresariales, se invita a incluir los resultados de los estudios de valoración de la calidad del servicio que tienen en cuenta las variables demográficas, donde se integren y se evalúe el impacto de las acciones de marketing en los segmentos del mercado, previamente identificados.

Conflicto de intereses: Este manuscrito fue preparado y revisado con la participación de todos los autores, quienes declaramos que no existe conflicto de intereses que ponga en riesgo la validez de los resultados presentados.

\section{BIBLIOGRAFÍA}

1. AFZAL, N.; PAKISTAN, S. 2013. Impact of customer satisfaction and demographic factors on customer' $S$ loyalty in banking sector of Pakistan. Middle-East $\mathrm{J}$. Scientific Res (Pakistan). 18(5):721-727.

2. AKBAR, S. 2013. Determinants of service quality and customer loyalty through the moderating effect of socio demographic characteristics. International J. Hospitality \& Tourism Systems (India). 6(2):81-91.

3. ANAND, S.V.; SELVARAJ, M. 2012. Impact of demographic variables on customer satisfaction in banking sector - an empirical study. International J. Scientific Res. Publications (Francia). 2(5):1-7.

4. ANIC, I.D.; RADAS, S. 2006. The role of satisfaction and demographic factors in building store loyalty. Ekonomska Politika (Croacia). 1(108):67-86.

5. BEAUDRY, J.S.; MILLER, L. 2016. Research literacy: a primer for understanding and using research. Guilford Publications (EEUU). 380p.

6. BELANCHE, D.; CASALÓ, L.V.; ORÚS, C. 2016. City attachment and use of urban services: benefits for smart cities. Cities (EEUU). 50:75-81.

7. BHATTACHARYA, S.; DASH, M.K. 2013. Measurement of customer satisfaction on demographic variables of banking sector in national capital region - an empirical analysis. Prestige International J. Management \& It- Sanchayan (India). 2(2):78-107.

8. BOLTON, N.; JAMES, H.D. 1991. A longitudinal analysis of the impact of service changes on customer attitudes. J. Marketing (EEUU). 55(1):1-9.

9. BRADBURN, N.; SUDMAN, S.; WANSINK, B. 2004. Asking questions: the definitive guide to questionnaire design - for market research, political polls, and social and health questionnaires. John Wiley \& Sons (EEUU). 448p.

10. CALABRESE, A.; COSTA, R.; ROSATI, F. 2016. Gender differences in customer expectations and perceptions of corporate social responsibility. J. Cleaner Production. (Países Bajos). 116(1):135-149.

11. CRONIN, J.J.; TAYLOR, S.A. 1992. Measuring service qualit: a reexamination and extension. J. Marketing (EEUU). 56(3):55-68.

12. DAVID, M.; SUTTON, C.D. 2011. Social research: an introduction. Sage Publications. (Inglaterra). 680p.

13. FINNEY, S.; DISTEFANO, C. 2006. Non-normal and categorical data in structural equation modeling. En: Hancock, G.R.; Mueller, R.O. (eds.). Structural Equation Modeling: a second course in structural equation modeling. Information Age (EEUU). p.269-312.

14. GANESAN-LIM, C.; RUSSELL-BENNETT, R.; DAGGER, T. 2008. The impact of service contact type and demographic characteristics on service quality perceptions. J. Services Marketing (Inglaterra). 22(7):550561. 
15. HAGAN, E. 2015. Service quality perceptions and sociodemographic characteristics of hotel guests in the western region of Ghana. J. Tourism Hospitality and Sport (Inglaterra). 10(1):16-30.

16. HAIR Jr, J.F.; BLACK, W.C.; BABIN, B.J.; ANDERSON, R.E. 2014. Multivariate data analysis. Pearson Education Limited (Inglaterra). 899p.

17. HARDY, M.A. 1993. Regression with dummy variables. Sage Publications (Inglaterra). 91p.

18. IRSHAD, R.; HASHMI, M.; HASSAN, A.; ZAHID, T.; HASSAN, S. 2013. Demographic variables impact on service climate and overall customer perception about service quality: evidence from healthcare industry. World Applied Sciences J. (Pakistan). 26(6):737-743.

19. JAIN, P. 2013. Service assurance perception and customer demography: empirical study of state bank of India. Global J. Management and Business Studies (India). 3(7):689-694.

20. JUWAHEER, T.D. 2011. Gender bias in hotel guests' perceptions of service quality: an empirical investigation of hotels in Mauritius. E-Review of Tourism Res. (EEUU). 9(5):164-189.

21. KHOSROW-POUR, M. 2012. Cases on assessment and evaluation in education. IGI Global (EEUU). 669p.

22. LADHARI, R.; LECLERC, A. 2013. Building loyalty with online financial services customers: is there a gender difference? J. Retailing Consumer Services (Inglaterra). 20(6):560-569.

23. LAVRAKAS, P.J. 2008. Encyclopedia of survey research methods. Sage Publications (Inglaterra). 1000p.

24. LEE, J.H.; KIM, H.D.; KO, Y.J.; SAGAS, M. 2011. The influence of service quality on satisfaction and intention: a gender segmentation strategy. Sport Management Review (Australia). 14(1):54-63.

25. MARKOVIĆ, S.; JANKOVIĆ, S.R. 2013. Exploring the relationship between service quality and customer satisfaction in croatian hotel industry. Tourism Hospitality Management (Croacia). 19(2):149-164.

26. MARUVADA, D.P.; BELLAMKONDA, R.S. 2013. Effects of demographic variables on railway passenger service quality: finding from Indian railway passenger service through rail qual. Int. J. Advances in Management and Economics (India). 2(5):159-163.
27. MELNYK, B.M.; MORRISON-BEEDY, D. 2012. Intervention research: designing, conducting, analyzing, and funding. Springer Publishing Company (EEUU). 496p.

28. MEYERS-LEVY, J.; LOKEN, B. 2015. Revisiting gender differences: what we know and what lies ahead. Journal of Consumer Psychology (EEUU). 25(1):129-149.

29. MIN, S.; KHOON, C.C. 2014. Demographic factors in the evaluation of service quality in higher education: A Structural Equation Model (Sem) Approach. Int. J. Marketing Studies (Canadá). 6(1):994-1010.

30. MITCHELL, M.L.; JOLLEY, J.M. 2010. Research design explained. Wadsworth Cengage Learning (EEUU). $645 p$.

31. MITCHELL, M.L.; JOLLEY, J.M. 2012. Research design explained. Wadsworth Cengage Learning (EEUU). $723 p$.

32. MOKHLIS, S. 2012. The influence of service quality on satisfaction: a gender comparison. Public Administration Research (Canadá). 1(1):103-112.

33. MONETTE, D.R.; SULLIVAN, T.J.; DEJONG, C.R. 2014. Applied social research: a tool for the human services. Brooks/Cole Cengage Learning (EEUU). 552p.

34. MORTON, C.; CAULFIELD, B.; ANABLE, J. 2016. Customer perceptions of quality of service in public transport: evidence for bus transit in Scotland. Case Studies on Transport Policy (Países Bajos). 4(3):199207.

35. MUTLAQ, N.; OTAIBI, A. 2014. Psychographic factors influence Saudi consumer's service quality in hypermarket. Int. J. Learning \& Development (EEUU). 4(4):97-113.

36. NETTLETON, D. 2003. Análisis de datos comerciales. Ediciones Díaz De Santos (España). 195p.

37. PALLI, J.G.; MAMILLA, R. 2012. Students' opinions of service quality in the field of higher education. Creative Education (EEUU). 3(4):430-438.

38. PARASURAMAN, A.; ZEITHAML, V.A.; BERRY, L.L. 1988. Servqual: a multiple-item scale for measuring consumer perceptions of service quality. Journal of Retailing (Inglaterra). 64(1):12-40. 
39. PATTERSON, P.G. 2007. Demographic correlates of loyalty in a service context. J. Services Marketing (Inglaterra). 21(2):112-121.

40. RAMEZ, W.S. 2011. Customers' socio-economic characteristics and the perception of service quality of Bahraini commercial banks. International J. Business and Management (Canadá). 6(10):113-126.

41. RANDOLPH, K.A.; MYERS, L.L. 2013. Basic statistics in multivariate analysis. Oxford University Press (Inglaterra). 224p.

42. RUBIN, A. 2013. Statistics for evidence-based practice and evaluation. Brooks/Cole Cengage Learning (EEUU).350p.

43. SAAD, R.; ISHAK, H.; JOHARI, N.R. 2013. The impact of demographic factors toward customer loyalty: a study on credit card users. Marketing Management (India). 55(1):13078-13084.

44. SALVADOR, C. 2007. Diferencias de género en la percepción de la calidad del servicio. Un estudio exploratorio. Boletín de Psicología (España). 89(1):29-45.

45. SASIKALA, D. 2013. The impact of service quality on customer satisfaction and loyalty in Indian banking sector: an empirical study through Servperf. Asia Pacific J. Marketing \& Management Review (India). 2(6):151-163.

46. SINGH, A. 2013. Demographic variables as antecedents of service quality and purchasing intention aspects in retailing. Global J. Management and Business Res. Accounting and Auditing (EEUU). 13(3):61-67.

47. STEENKAMP, J.B.E.M.; VAN TRIJP, H.C.M. 1991. The use of Lisrel in validating marketing constructs. Int. J. Res. Marketing (Países Bajos). 8(4):283-299.

48. TOLPA, E. 2012. Measuring customer expectations of service quality: case airline industry. Aalto University School of Economics (Finlandia). 95p.
49. VERGARA-SCHMALBACH, J.C.; QUIESADA, V.M. 2011. Análisis de la calidad en el servicio y satisfacción de los estudiantes de ciencias económicas de la universidad de Cartagena mediante un modelo de ecuaciones estructurales. Redie (México). 13(1):108-122.

50. VERGARA-SCHMALBACH, J.C.; QUESADA, V.M.; BLANCO, I. 2011. Análisis de la calidad en el servicio y satisfacción de los usuarios en dos hoteles cinco estrellas de la ciudad de Cartagena (Colombia) mediante un modelo de ecuaciones estructurales. IngeniareRev. Chilena Ingeniería (Chile). 19(3):420-428.

51. VERGARA-SCHMALBACH, J.C.; QUESADA, V.M.; BLANCO, I. 2012. Factores clave para la valoración de la calidad del servicio y satisfacción del cliente: modelos causales, desarrollo y evolución. Rev. Virtual Universidad Católica Del Norte (Chile). 35(1):380-400.

52. WEDEL, M.; DESARBO, W.S. 2002. Market segment derivation and profiling via a finite mixture model framework. Marketing Letters (Países Bajos). 13(1):1725.

53. YOSHIDA, K. 2011. Gender perceptions in southeast asian countries: findings from Jica-Ri value surveys. Japan Int. Cooperation Agency (Japan). 13p.

54. ZALATAR, W.F. 2012. Quantifying customers' gender effects on service quality perceptions of Philippine commercial banks. Procedia - Social Behavioral Sciences (Inglaterra). 57(9):268-274.

55. ZENG, C.; NIU, D.; Li, H.; ZHOU, T.; ZHAO, Y. 2016. Public perceptions and economic values of sourceseparated collection of rural solid waste: a pilot study in China. Resources, Conservation And Recycling (Países Bajos). 107(1):166-173.

Recibido: Marzo 30 de 3017

Aceptado: Agosto 22 de 2017

Cómo citar:

Vergara Schmalbach, J.C.; Quesada Ibargüen, V.M.; Maza Ávila, F.J. 2017. Efecto de los aspectos demográficos en la valoración de la calidad del servicio. Una revisión. Rev. U.D.C.A Act. \& Div. Cient. 20(2): 457-467. 